\title{
Nível de ácido úrico como biomarcador diagnóstico e prognóstico de doenças cardiovasculares
}

\author{
Uric acid level as a diagnostic and prognostic biomarker of \\ cardiovascular diseases
}

\author{
Venícius Djalma da Rosa ${ }^{1}$; Thiago Bordinhão²; Juliana Buck Dias ${ }^{3}$; Alessandra \\ Miyuki Okino ${ }^{4}$; Danielle Venturini ${ }^{5}$
}

\begin{abstract}
Resumo
Diversos estudos propõem a relação entre hiperuricemia e doenças cardiovasculares, implicando em uma relação causal, aumento da morbimortalidade cardiovascular e melhoria da evolução clínica, por meio de farmacoterapia para redução de níveis de ácido úrico. A despeito destes achados, ainda incertos, o que a maioria das evidências sugere é que níveis de ácido úrico podem representar um biomarcador de diagnóstico e/ou prognóstico útil e barato. Portanto, a presente revisão de literatura apresenta as principais e mais recentes evidências sobre a relação de ácido úrico com doenças cardiovasculares, tais como aterosclerose, síndrome metabólica, doença arterial coronariana, insuficiência cardíaca, hipertensão, infarto agudo do miocárdio e mortalidade por doenças cardiovasculares. Os dados mais controversos dizem respeito à doença arterial coronariana, possivelmente por tratar-se de uma síndrome que contemple duas condições - angina instável e infarto agudo do miocárdio - tornando a análise pouco específica. A maioria dos estudos sugere que o ácido úrico, principalmente a hiperuricemia, seja um bom preditor diagnóstico (hipertensão e infarto agudo do miocárdio) e prognóstico (doença arterial coronariana e insuficiência cardíaca), no entanto poucos estudos avaliaram o desempenho do marcador em termos de curva ROC, sendo impossível emitir conclusões bem fundamentadas neste sentido. Considerando os resultados da maioria das evidências, a acessibilidade e o baixo custo da mensuração dos níveis de ácido úrico, é recomendável a integração da avaliação da hiperuricemia em um conjunto de análise de outros fatores de risco.
\end{abstract}

Palavras-chave: Hiperuricemia. Hipertensão. Doenças cardiovasculares.

\begin{abstract}
Several studies have suggested an association between hyperuricemia and cardiovascular diseases, resulting in a causal relationship, increased cardiovascular morbidity and improving clinical outcomes by means of pharmacotherapy for reducing uric acid levels. Though still uncertain, most studies suggest that levels of uric acid might be a useful and inexpensive biomarker for diagnosis and / or prognosis. Therefore, this literature review presents the most recent major evidence of uric acid related to cardiovascular diseases such as atherosclerosis, metabolic syndrome, coronary artery disease, heart
\end{abstract}

${ }^{1}$ Farmacêutico, Especialista em Análises clínicas pela Universidade Estadual de Londrina (UEL), Londrina-PR.

${ }^{2}$ Farmacêutico, Residente em Análises Clínicas, Departamento de Patologia, Análises Clínicas e Toxicológicas da Universidade Estadual de Londrina (UEL), Londrina-PR.

${ }^{3}$ Farmacêutica e Residente em Análises Clínicas, Departamento de Patologia, Análises Clínicas e Toxicológicas da Universidade Estadual de Londrina (UEL), Londrina-PR.

${ }^{4}$ Farmacêutica, Mestre em Análises clínicas, Docente do Centro de Ciências da Saúde, Departamento de Patologia, Análises Clínicas e Toxicológicas, Universidade Estadual de Londrina (UEL), Londrina-PR.

${ }^{5}$ Farmacêutica, Doutora em Ciências da Saúde, Docente do Centro de Ciências da Saúde, Departamento de Patologia, Análises Clínicas e Toxicológicas, área bioquímica clínica, Universidade Estadual de Londrina (UEL), Londrina-PR. 
failure, hypertension, myocardial infarction and mortality from cardiovascular disease. The most controversial data refer to coronary artery disease, possibly because it is a syndrome that includes two conditions - unstable angina and acute myocardial infarction - which makes the analysis low in specificity. Several articles have demonstrated that uric acid, especially hyperuricemia, is a good diagnosis predictor (hypertension and acute myocardial infarction) and prognosis (coronary artery disease and heart failure), however few studies have evaluated the performance of the marker in terms of ROC curve, making it impossible to deliver well-founded conclusions in this regard. Considering the results of most of the evidence, accessibility and low cost of measurements of uric acid levels, we recommend to integrate both the evaluation of hyperuricemia and the analysis of other risk factors.

Keywords: Hyperuricemia, Hypertension, Cardiovascular diseases.

\section{Introdução}

Hiperuricemia, depósito de urato e gota são condições relacionadas e comuns na população, decorrentes do acúmulo de ácido úrico (AU), produto final do catabolismo de nucleotídeos purínicos. Hiperuricemia é definida por um nível plasmático de $\mathrm{AU}$ maior que $6,8 \mathrm{mg} / \mathrm{dL}$ à temperatura fisiológica $\left(37^{\circ} \mathrm{C}\right)$ e $\mathrm{pH}$ neutro. No entanto, outras evidências apontam para a necessidade de adaptar a faixa para $6,0 \mathrm{mg} / \mathrm{dL}$ tratando-se de pacientes com hiperuricemia crônica ou gota, a fim de evitar depósitos de cristais de AU (GRASSI et al., 2013). Depósitos de uratos podem estar presentes na hiperuricemia assintomática, contudo quando se manifesta clinicamente apresenta-se como artrites deformativas e erosivas (manifestações articulares), nefrolitíase e nefropatia crônica (manifestações extra-articulares) (GRASSI et al., 2013). Por outro lado, a gota cursa com a presença de depósitos de uratos e corresponde a uma artrite inflamatória aguda causada pela intensa inflamação secundária à deposição crônica de cristais de urato monossódico em articulações (GRASSI et al., 2013).

A formação de AU ocorre a partir da quebra de adenosina e guanina (purinas) via hipoxantina à xantina e então a AU, por meio da xantina oxidase (GRASSI et al., 2013; KANELLIS; FEIG; JOHNSON, 2004). As reações catalisadas por xantina oxidase geram espécies reativas de oxigênio como subprodutos, tais como o ânion superóxido que reage com prótons e óxido nítrico para originar novas espécies reativas de oxigênio. Essas, por sua vez, promovem lesão do endotélio cardiovascular e da microvasculatura (KAUFMAN; GUGLIN, 2013).

Este processo pode ser um dos responsáveis pela relação proposta por diversos estudos entre hiperuricemia e doenças cardiovasculares (FANG; ALDERMAN, 2000; FEIG; KANG; JOHNSON, 2008; GRASSI et al., 2013; KANELLIS; FEIG; JOHNSON, 2004; KIVITY et al., 2013; KRISHNAN; SOKOLOVE, 2011; NISKANEN et al., 2014). Tal associação pode implicar em uma relação causal (KRISHNAN; SOKOLOVE, 2011) e em aumento da mortalidade cardiovascular (FANG; ALDERMAN, 2000; NISKANEN et al., 2014). Outras evidências indicam ainda melhoria da evolução clínica das doenças cardiovasculares a partir da inserção de agentes redutores dos níveis de AU, tais como alopurinol e febuxostat (FARQUHARSON et al., 2002; HARE et al., 2008; NASR; MAURICE, 2010; GRASSI et al., 2013; KAUFMAN; GUGLIN, 2013; YANIK; FEIG, 2013).

Com relação às doenças cardiovasculares e a despeito dos benefícios da farmacoterapia de hiperuricemia, da possível relação causal ou do aumento da morbimortalidade, o que a maioria das evidências sugere é que níveis séricos de $\mathrm{AU}$ podem representar um biomarcador de diagnóstico e/ou prognóstico útil e barato. Portanto, a presente revisão de literatura irá apresentar as principais e mais recentes evidências sobre a relação de AU com doenças cardiovasculares. 


\section{Desenvolvimento}

\section{Aterosclerose e síndrome metabólica}

Wannamethee et al. (1997), em uma coorte realizada com 7.688 homens, identificaram a relação entre AU e o risco de doença cardíaca coronária a depender de presença de infarto do miocárdio pré-existente e aterosclerose generalizada. Desta forma, concluíram que AU não é um fator de risco independente para a doença arterial coronariana (DAC), mas pode compor um conjunto de fatores de risco associados com síndrome metabólica (SM) - condição que inclui intolerância à glicose, dislipidemia, obesidade central e pressão sanguínea elevada.

Muitas evidências apontam para a relação entre nível de AU e doenças cardiovasculares avançadas. No entanto, pouco se sabe se há relação também com fases iniciais da aterosclerose, demonstradas pela espessura da camada íntima-média carótida, placa de carótida, distensibilidade carotídea e dilatação fluxo-mediada da artéria braquial. Portanto, Oikonen et al. (2012) examinaram 1.985 jovens adultos e identificaram que níveis séricos elevados de AU estão associados com marcadores de risco cardiovascular em adultos jovens, especialmente com o índice de massa corporal, mas não identificaram evidência de que o mesmo desempenharia papel independente na fisiopatologia da aterosclerose precoce.

Pacifico et al. (2009) em estudo realizado na Itália com o objetivo de verificar a associação da hiperuricemia com a síndrome metabólica e aterosclerose em crianças e adolescentes obesos, concluíram que pacientes com elevadas concentrações de ácido úrico tiveram incidência maior da aterosclerose carotídea avaliada através do espessamento do complexo médio-intimal da carótida pela ultrassonografia com dopler nesta região.

A importância da hiperuricemia como causa da SM apresenta várias implicações terapêuticas. O maior risco de morte por todas as causas e de DCV associada à hiperuricemia está bem estabelecida e não há dúvida de que a hiperuricemia prediz o desenvolvimento de hipertensão, disfunção endotelial e SM. (SIMÃO, LOZOVOY, DICHI, 2012).

\section{Doença arterial coronariana e circulação colateral coronariana}

A doença arterial coronariana (DAC) e sua relação com AU é a mais controversa dentre as doenças cardiovasculares. Uysal et al. (2014) avaliaram 480 pacientes consecutivos com DAC estável e identificaram que níveis de glicemia de jejum, leucócitos, creatinina, contagem de plaquetas e AUS foram significativamente maiores em pacientes com desenvolvimento pobre de circulação colateral coronária (CCC) do que naqueles com boa CCC. No entanto, o corte de $5,65 \mathrm{mg} / \mathrm{dL}$ para AUS apresentou sensibilidade de $60 \%$ e especificidade de $66 \%$ através de análise de curvas ROC.

Kasapkara et al. (2012) avaliaram 175 pacientes com síndrome coronariana aguda sem supradesnivelamento de segmento ST, os quais foram divididos em dois grupos, com base em níveis de AU: grupo I com níveis normais de AU e grupo II com níveis elevados de AU. Os autores identificaram que o grupo II teve uma taxa significativamente maior de $\mathrm{CCC}$ pouco desenvolvida e uma menor taxa de CCC bem desenvolvida em comparação com o grupo I ( $p=$ 0,003 e 0,001 , respectivamente). Pacientes com má CCC tinham níveis significativamente mais elevados de AUS em comparação com pacientes com CCC bem desenvolvida $(6,5 \pm 1,1$ vs $5,5 \pm$ $1,7 \mathrm{mg} / \mathrm{dL}, \mathrm{p}=0,028)$. Análises de regressão linear mostraram que o desenvolvimento pobre de CCC foi significativamente associado com os níveis séricos de $\mathrm{AU}$ (coeficiente $=0,22, \mathrm{p}=0,005$ ).

Krishnan et al. (2012) avaliaram a presença de DAC em pacientes com infarto agudo do miocárdio (IAM) prévio. Para isso, avaliaram 4.352 
participantes e observaram por meio de regressão multivariada que os desfechos mortalidade por todas as causas (taxa de risco (HR) 1,88; IC 95\%, 1,45-2,46), mortalidade por DAC (HR 1,99; IC $95 \%, 1,49-2,66)$ e incidência de IAM (1,36; IC 95\%, 1,08-1,70) foram maiores no quarto quartil de AU. Além disso, os participantes com gota não tratada tinham uma razão de risco ajustada de 1,5 a 2,0 (p $<0,01)$ para estes desfechos, em contrapartida dos participantes com gota tratada, demonstrando que AU pode ser um marcador prognóstico independente para mortalidade por todas as causas e por DAC em pacientes com IAM recente.

Ndrepepa et al. (2013) incluíram 3.705 pacientes com diabetes mellitus e DAC. Das 264 mortes que ocorrem durante o acompanhamento, 45 foram em pacientes do primeiro quartil de $\mathrm{AU}, 43$ mortes em pacientes do segundo quartil de AU, 51 mortes em pacientes do terceiro quartil de $\mathrm{AU}$ e 125 mortes em pacientes do quarto quartil de $\mathrm{AU}$, com estimativas de Kaplan-Meier da mortalidade de 5,1, 4,8, 5,6 e $14,0 \%$, respectivamente; taxa de risco não ajustada, 2,81 (IC 95\%, 2,21-3,58; $\mathrm{p}<0,001$ ) para o quarto quartil em comparação com primeiro ao terceiro quartis. Em conclusão, em pacientes com Diabetes mellitus tipo 2 e DAC confirmada, níveis elevados de AU preveem a mortalidade independentemente de conhecidos fatores de risco cardiovascular.

Desta forma, estes quatro estudos indicam a utilização do nível sérico de AU como biomarcador útil para a estratificação de risco (marcador prognóstico independente e como preditor de mortalidade).

Contudo, estudos anteriores (CULLETON et al., 1999; WHEELER et al., 2005) apontam para resultados discordantes. No estudo de 1999, foi analisada a relação com 6.763 participantes do Framingham Heart Study (idade média de 47 anos) e identificaram: nos homens, após ajuste para idade, que o nível de AU elevado não foi associado com aumento do risco de um resultado adverso; enquanto nas mulheres, após ajuste para idade, nível de AU foi preditivo de doença arterial coronariana $(\mathrm{p}=0,002)$, morte por doença cardiovascular $(\mathrm{p}=$ $0,009)$ e morte por todas as causas $(p=0,03)$. Após o ajuste para fatores de risco adicional para doenças cardiovasculares, o nível de AU não foi mais associado à DAC, morte por doença cardiovascular ou morte por todas as causas. Os autores concluem, portanto, que o $\mathrm{AU}$ não tem um papel causal no desenvolvimento de doença coronariana, morte por doença cardiovascular ou morte por todas as causas.

Wheeler et al. (2005) corroboram com esses dados, indicando que em comparação aos indivíduos no terço inferior do baseline de $\mathrm{AU}$, aqueles no terço superior tinham uma relação de 1,39 (IC 95\%, $1,23-1,58$ ) que caiu para 1,12 (IC 95\% 0,97-1,30) após ajuste para tabagismo e outros fatores de risco. Os mesmos autores realizaram ainda meta-análise de 16 estudos prospectivos e identificaram um odds ratio de 1,13 (IC 95\%, 1,07-1,20) que caiu para 1,02 (IC 95\%, 0,91-1,14) quando incluídos apenas os estudos com ajuste completo para possíveis fatores de confusão.

Dessa forma, ambos os estudos concluem ser improvável que AU seja um dos principais determinantes da doença e sugerem que a associação identificada em outros estudos deva-se à associação do nível de AU com outros fatores de risco.

\section{Hipertensão arterial sistêmica}

A hipertensão arterial sistêmica é uma das condições mais estudadas com relação ao AU. Taniguchi et al. (2001) com o objetivo de investigar a associação de nível de AU com o risco para hipertensão e diabetes tipo 2, realizaram uma coorte prospectiva com 6.356 homens japoneses e identificaram que nível de AU foi associado com maior risco de hipertensão, mas não para diabetes tipo 2. Depois de ajustar para conhecidos fatores de risco, incluindo consumo diário de álcool, o nível sérico de AU foi associado com um aumento para o risco de hipertensão; o risco relativo para hipertensão foi 1,00 para quintil 1 do nível sérico de 
AU, 1,24 (IC 95\%, 0,94-1,65) para quintil 2, 1,34 (IC 95\%, 1,03-1,76) para quintil 3, 1,76 (IC 95\%, $1,35-2,29$ ) para quintil 4 e 2.01 (IC 95\% 1,56-2,60) para quintil $5(\mathrm{p}<0,001)$.

Nagahama et al. (2004) analisaram prospectivamente a relação entre hiperuricemia e o risco de desenvolvimento de hipertensão em um total de 4.489 indivíduos sem hipertensão. Ao final do estudo, a análise multivariada foi desenvolvida com ajuste para idade, história de hipertensão familiar, consumo de álcool, tabagismo, obesidade, hipercolesterolemia, hipertrigliceridemia, HDLcolesterol e Diabetes mellitus e identificaram um odds ratio (IC 95\%) em homens de 1,48 (1,08-2,02) e em mulheres de $1,90(1,03-3,51)(\mathrm{p}<0,05)$.

Os resultados destes estudos são corroborados por outros 16 estudos identificados em revisão (FEIG; KANG; JOHNSON, 2008), que indicam um risco relativo de hipertensão de 1,1 a 2,0 vezes maiores a depender do nível elevado de $\mathrm{AU}$, independente de outros fatores de risco.

Com relação às crianças, Yanik e Feig (2013) estudos têm demonstrado uma associação entre hiperuricemia e hipertensão arterial, corroborando com o uso do nível sérico como um biomarcador para o diagnóstico.

\section{Insuficiência cardíaca}

Foram identificados três estudos que apresentam os benefícios da farmacoterapia com alopurinol e oxipurinol (metabólito ativo de alopurinol) em insuficiência cardíaca aguda e crônica. Farquharson et al. (2002) propõem que o aumento do estresse oxidativo na insuficiência cardíaca crônica contribua para a disfunção endotelial, por meio da ação da xantina oxidase. Portanto, os autores testaram alopurinol e identificaram em 11 pacientes com insuficiência cardíaca crônica por meio de ensaio clínico randomizado, cruzado, duplocego e controlado por placebo, e concluíram que o alopurinol melhora a disfunção endotelial na insuficiência cardíaca crônica, podendo reduzir eventos cardiovasculares e até mesmo melhorar a capacidade de exercício na insuficiência cardíaca crônica.

Nasr e Maurice (2010) também avaliaram os benefícios de alopurinol em pacientes $(n=59)$ com insuficiência cardíaca crônica, contra placebo. Numa análise de subgrupo, os pacientes com elevado AU (mais de $7 \mathrm{mg} / \mathrm{dL}$ ) responderam favoravelmente ao alopurinol enquanto aqueles com $\mathrm{AU}$ menor que $7 \mathrm{mg} / \mathrm{dL}$ exibiram uma tendência de nenhuma mudança. Além disso, a redução AU por alopurinol foi correlacionada com resposta clínica e funcional favoráveis.

Hare et al. (2008) avaliaram o benefício de oxipurinol em 405 pacientes randomizados para oxipurinol ou placebo. As porcentagens de pacientes caracterizados como melhora, inalterado ou piora, não diferiram entre os que receberam oxipurinol ou placebo. No entanto, em uma análise de subgrupo, os pacientes com AU elevado $(>9,5 \mathrm{mg} / \mathrm{dL}, \mathrm{n}=$ 108) responderam favoravelmente ao oxipurinol $(p=0,02)$, enquanto os pacientes tratados com oxipurinol com $\mathrm{AU}<9,5 \mathrm{mg} / \mathrm{dL}$ apresentaram uma tendência para o agravamento. Desta forma, oxipurinol não promoveu melhora clínica em pacientes com insuficiência cardíaca moderada a grave, mas naqueles com níveis elevados de AU.

Tanto Nasr e Maurice, quanto Hare et al. concluíram que alopurinol e oxipurinol não produziram melhoras clínicas em pacientes com insuficiência cardíaca moderada a grave, mas apenas naqueles com níveis elevados de AU.

Os três estudos apontam para o benefício da farmacoterapia em insuficiência cardíaca crônica, o que permitiria deduzir que haja relação entre hiperuricemia e a patogenia da doença. No entanto, em revisão recente (KAUFMAN; GUGLIN, 2013) concluiram que apesar do AU ser um marcador independente de prognóstico adverso na IC aguda e crônica, não há evidência que suporte uma relação com a patogênese de insuficiência cardíaca crônica. 


\section{Infarto agudo do miocárdio}

Ácido úrico é um importante antioxidante, responsável por $60 \%$ da remoção de radicais livres presentes no plasma atuando como removedor (scavenging) de espécies reativas de oxigênio e nitrogênio (EROs/ERNs). Em um estudo prévio, foi observado que pacientes com SM mostraram aumento do estresse oxidativo (EO), enquanto a capacidade antioxidante total, avaliada por um método que detecta antioxidantes hidrossolúveis presentes no plasma através da metodologia total radical-trapping antioxidant parameter (TRAP), não diminuiu neste estudo, provavelmente pela presença de níveis séricos elevados de AU (SIMÃO; DICHI; BARBOSA, 2008).

Por outro lado, um estudo conduzido por nosso grupo, avaliou a influência da SM na oxidação lipídica e protéica e nas defesas antioxidantes em pacientes com sobrepeso com e sem SM e indivíduos controle. Foi observado que o grupo com SM quando comparado aos grupos sobrepeso sem SM e controle apresentou níveis elevados de triacilglicerol, glicose, insulina, homeostasis model assessment - insulin resistance (HOMA-IR) e AU. Como os indivíduos com SM apresentavam hiperuricemia, os níveis de TRAP foram corrigidos pelos níveis séricos de AU e evidenciou que esses indivíduos apresentavam menores valores de TRAP quando comparados aos outros dois grupos, mostrando efetivamente a participação do AU como antioxidante (VENTURINI et al., 2012).

AU age como antioxidante apenas em ambientes hidrofílicos, o que representa a maior limitação do AU como antixidante, não sendo capaz de remover radicais como ânion superóxido. Ele pode agir como prooxidante em condições de EO, como encontrado na SM (SIMÃO, LOZOVOY, DICHI, 2012).

Tendo em vista que o AU é um marcador de EO e de hipóxia, a qual precede necrose de cariomiócitos no IAM, o estudo de Wildi et al. (2013) avaliaram o potencial de AU como marcador útil para diagnóstico precoce e estratificação de rico de pacientes com suspeita de IAM. Para isso, realizaram um estudo observacional com 892 pacientes consecutivos que foram acompanhados por 24 meses. O AU na apresentação foi maior nos pacientes com IAM que em pacientes sem $(372 \mu \mathrm{M}$ vs. $336 \mu \mathrm{M} ; \mathrm{p}<0,001)$. A acurácia do diagnóstico de AU para IAM quantificada pela área sobre a curva ROC foi de 0,60 (IC 95\% 0,56 - 0,65). Quando adicionado a troponina $\mathrm{T}$, AU significativamente aumentou a AUC ROC de troponina T de 0,89 (IC $95 \% 0,85$ - 0,93) para 0,92 (IC 95\% 0,89-0,95, p $=0,020)$. As taxas cumulativas de mortalidade de 24 meses foram $2,2 \%$ no primeiro, $5,4 \%$ no segundo e no terceiro e $15,6 \%$ no quarto quartil de $\mathrm{AU}$ ( $\mathrm{p}<$ $0,001)$. O AU predisse a mortalidade de 24 meses de forma independente.

Já entre pacientes com IAM prévio, o uso de AU parece ter seu valor para predizer o fluxo sanguíneo coronariano em pacientes submetidos à intervenção percutânea coronariana, como identificado por Akpek et al. (2011).

Desta forma, ambos os estudos apontam para a utilidade do AU no diagnóstico precoce e na estratificação de risco de pacientes com suspeita de IAM (WILDI et al., 2013) e para a estratificação de risco em pacientes com IAM com supra de ST, podendo contribuir para implicações terapêuticas (AKPEK et al., 2011).

\section{Mortalidade por doenças cardiovasculares}

Corroborando para a utilidade de prognóstico e de estratificação de risco de AU, alguns estudos identificaram associação entre níveis séricos de AU e mortalidade por doenças cardiovasculares.

Fang e Alderman (2000) acompanharam 5.926 indivíduos, por um período médio de 16,4 anos, com idades entre 25 e 74 anos e observaram que o aumento dos níveis séricos de AU teve uma relação positiva com a mortalidade cardiovascular em homens e mulheres e em pessoas negras e brancas. 
Mortes por doença cardíaca isquêmica em homens e mulheres aumentou quando os níveis séricos de AU estavam no quartil mais elevado em comparação com o quartil mais baixo ( $>416$ homens vs $<321$ $\mu \mathrm{mol} / \mathrm{L}$, relação de risco, 1,77 [IC 95\%, 1,08-3,98]; mulheres, $>333$ vs. $<238 \mu \mathrm{mol} / \mathrm{L}$, relação de risco, 3,00 [IC 95\%, 1,45-6,28]). As razões de risco para os homens foram 1,09 (IC 95\%, 1,02-1,18) e 1,17 (IC 95\%, 1,06-1,28), e para as mulheres eram 1,26 (IC 95\%, 1,16-1,36) e 1,30 (IC 95\%, 1,17- 1,45), respectivamente, após o ajuste para idade, raça, índice de massa corporal, tabagismo, consumo de álcool, nível de colesterol, história de hipertensão e diabetes, e uso de diuréticos.

Ao encontro desta associação, uma coorte prospectiva (NISKANEN et al., 2014) com acompanhamento médio de 11,9 anos de 1.423 homens sem doença cardiovascular, câncer ou diabetes, foi identificado por meio de análises ajustadas para idade, que os níveis séricos de AU no terço superior foram associados com um risco de 2,5 vezes maior de morte por doença cardiovascular do que os níveis no terço inferior, demonstrando que o nível de AU foi um forte preditor de mortalidade por doença cardiovascular em homens de meiaidade saudáveis.
Em outro estudo, (CHOI; CURHAN, 2007) 51.297 homens foram avaliados por um período de mais de 12 anos. Em comparação com os homens sem histórico de gota e DAC no início do estudo, os riscos relativos multivariados entre os homens com história de gota foram 1,28 (IC 95\%, 1,15$1,41)$ para a mortalidade total, 1,38 (IC 95\%, 1,151,66) para mortes por doença cardiovascular, e 1,55 (IC 95\%, 1,24-1,93) para DAC fatal. Os riscos relativos correspondentes entre os homens com DAC preexistente foram 1,25 (IC 95\%: 1,09-1,45), 1,26 (IC 95\%, 1,07 a 1,50) e 1,24 (IC 95\%, 1,04$1,49)$, respectivamente. Além disso, homens com gota tinham um risco maior de infarto do miocárdio não fatal do que os homens sem gota (risco relativo multivariado, 1,59, IC 95\%, 1,04-2,41). Dessa forma, os homens com gota têm um maior risco de morte por todas as causas. Entre os homens sem DAC pré-existente, o aumento do risco de mortalidade é principalmente um resultado de um risco elevado de morte por doença cardiovascular, particularmente de DAC. Tais evidências apontam para a importância de utilizar os níveis elevados de AU como marcador prognóstico. Na tabela 1 mostramos os principais estudos que avaliaram a relação dos níveis séricos de AU e doenças cardiovasculares.

Tabela 1 - Síntese qualitativa dos principais resultados de estudos que avaliaram relação entre ácido úrico e doenças cardiovasculares.

\begin{tabular}{|c|c|c|}
\hline Identificação & Condição/ doença & Principais resultados \\
\hline Oikonen et al. (2012) & Aterosclerose precoce & $\begin{array}{l}\text { Associação com marcadores de risco cardiovascular, mas não com fisiopatologia da } \\
\text { aterosclerose precoce. }\end{array}$ \\
\hline Wannamethee et al. (1997) & Aterosclerose e IAM pré-existente & AU não é um fator de risco independente para DAC. \\
\hline Uysal et al. (2014) & DAC & $\mathrm{AU}$ maior em pacientes com desenvolvimento pobre de $\mathrm{CCC}$. \\
\hline Kasapkara et al. (2012) & DAC & esenvolvimento pobre de $\mathrm{CCC}$ foi associado com $\mathrm{AU}$. \\
\hline Krishnan et al. (2012) & DAC e IAM prévio & $\begin{array}{l}\text { Mortalidade por todas as causas, mortalidade por DAC e incidência de IAM foram } \\
\text { maiores no quarto quartil de AU. }\end{array}$ \\
\hline Ndrepepa et al. (2013) & DAC e diabetes mellitus & Hiperuricemia prevê mortalidade independente de conhecidos fatores de risco. \\
\hline Culleton et al. (1999) & DAC & $\begin{array}{l}\text { Após ajuste para fatores de risco, o nível de } \mathrm{AU} \text { não foi associação à } \mathrm{DAC} \text {, morte por } \\
\text { doença cardiovascular ou morte por todas as causas. }\end{array}$ \\
\hline Wheeler et al. (2005) & DAC & Após ajuste para fatores de risco, ausência de associação entre AU e DAC. \\
\hline Taniguchi et al. (2001) & Hipertensão & Após ajuste para fatores de risco, $\mathrm{AU}$ foi associado com aumento do risco de hipertensão. \\
\hline Nagahama et al. (2004) & Hipertensão & $\begin{array}{l}\text { Após ajuste para fatores de risco, presença de associação entre hiperuricemia e o risco de } \\
\text { desenvolvimento de hipertensão. }\end{array}$ \\
\hline Yanik e Feig (2013) & Hipertensão & $\begin{array}{l}\text { Presença de associação entre hiperuricemia e o risco de desenvolvimento de hipertensão } \\
\text { essencial em crianças. }\end{array}$ \\
\hline Farquharson et al. (2002) & juficiência cardí & Alopurinol melhora a disfunção endotelial. \\
\hline Nasr e Maurice (2010) & Insuficiência cardíaca & Alopurinol produz melhorias clínicas em pacientes com níveis elevados de AUS. \\
\hline Hare et al. (2008) & Insuficiência cardíaca & Oxipurinol produz melhorias clínicas em pacientes com níveis elevados de AUS. \\
\hline Wild et al. (2013) & IAM suspeito & AU prediz a mortalidade de 24 meses de forma independente. \\
\hline Akpek et al. (2011) & IAM prévio & $\begin{array}{l}\text { AU prediz fluxo sanguíneo coronariano em pacientes submetidos a intervenção } \\
\text { percutânea coronariana. }\end{array}$ \\
\hline Fang e Alderman (2000) & Mortalidade por doença CDV & $\begin{array}{l}\text { Mortes por DCV aumentou quando os níveis séricos de } \mathrm{AU} \text { estavam no quartil mais } \\
\text { elevado em comparação com o quartil mais baixo. }\end{array}$ \\
\hline & & AU no terço superior foram associados com morte por DCV. \\
\hline Choi e Curhan (2007) & Mortalidade por doença CDV & Associaçăo entre AU e mortalidade total, mortalidade por DCV e DAC fatal. \\
\hline
\end{tabular}

Legenda: $\mathrm{AU}$ : ácido úrico; $\mathrm{CDV}$ : cardiovascular; DAC: doença arterial coronariana; IAM: infarto agudo do miocárdio.

Fonte: Autores. 


\section{Conclusão}

A presente revisão de literatura apresentou a associação entre hiperuricemia e aterosclerose, síndrome metabólica, doença arterial coronariana, insuficiência cardíaca, hipertensão, infarto agudo do miocárdio e mortalidade por doenças cardiovasculares.

Os dados mais controversos dizem respeito à doença arterial coronariana, possivelmente por tratar-se de uma síndrome que contemple duas condições - angina instável e infarto agudo do miocárdio -, tornando a análise pouco específica.

A maioria dos estudos sugere que o ácido úrico, principalmente em quadros de hiperuricemia, seja um bom preditor diagnóstico (hipertensão e infarto agudo do miocárdio) e prognóstico (doença arterial coronariana e insuficiência cardíaca), no entanto poucos estudos avaliaram o desempenho do marcador em termos de curva ROC, sendo impossível emitir conclusões bem fundamentadas neste sentido. Para resolução das controversas e preenchimento das lacunas, faz-se necessária a realização de mais estudos primários avaliando as características de desempenho dos marcadores quanto à sensibilidade, especificidade, valor preditivo positivo e negativo, bem como estudos secundários de maior força de evidência como revisões sistemáticas seguidas de meta-análise, que considerem qualidade metodológica, riscos de viés e heterogeneidade dos estudos primários, a fim de emitir conclusões robustas metodologicamente.

Considerando os resultados da maioria das evidências, a acessibilidade e o baixo custo da mensuração dos níveis deácidoúrico, érecomendável à integração da avaliação de hiperuricemia em um conjunto de análise de fatores de risco.

\section{Conclusão}

A presente revisão de literatura apresentou a associação entre hiperuricemia e aterosclerose, síndrome metabólica, doença arterial coronariana, insuficiência cardíaca, hipertensão, infarto agudo do miocárdio e mortalidade por doenças cardiovasculares.

Os dados mais controversos dizem respeito à doença arterial coronariana, possivelmente por tratar-se de uma síndrome que contemple duas condições - angina instável e infarto agudo do miocárdio -, tornando a análise pouco específica.

A maioria dos estudos sugere que o ácido úrico, principalmente em quadros de hiperuricemia, seja um bom preditor diagnóstico (hipertensão e infarto agudo do miocárdio) e prognóstico (doença arterial coronariana e insuficiência cardíaca), no entanto poucos estudos avaliaram o desempenho do marcador em termos de curva ROC, sendo impossível emitir conclusões bem fundamentadas neste sentido. Para resolução das controversas e preenchimento das lacunas, faz-se necessária a realização de mais estudos primários avaliando as características de desempenho dos marcadores quanto à sensibilidade, especificidade, valor preditivo positivo e negativo, bem como estudos secundários de maior força de evidência como revisões sistemáticas seguidas de meta-análise, que considerem qualidade metodológica, riscos de viés e heterogeneidade dos estudos primários, a fim de emitir conclusões robustas metodologicamente.

Considerando os resultados da maioria das evidências, a acessibilidade e o baixo custo da mensuração dosníveis deácidoúrico,érecomendável à integração da avaliação de hiperuricemia em um conjunto de análise de fatores de risco.

\section{Referências}

AKPEK, M.; KAYA M. G.; UYAREL, H.; YARLIOGLUES, M.; KALAY, N.; GUNEBAKMAZ, O.; DOGDU, O.; ARDIC, I.; ELCIK, D.; SAHIN, O.; OGUZHAN, A.; ERGIN, A.; GIBSON, C. M. The association of serum uric acid levels on coronary flow in patients with STEMI undergoing primary PCI. Atherosclerosis, Limerick, v. 219 , n. 1, p. 334-41, nov. 2011. 
CHOI, H. K.; CURHAN, G. Independent impact of gout on mortality and risk for coronary heart disease. Circulation, Baltimore, v. 116, n. 8, p. 894-900, aug. 2007.

CULLETON, B. F.; LARSON, M. G.; KANNEL, W. B.; LEVY, D. Serum uric acid and risk for cardiovascular disease and death: the Framingham Heart Study. Annals of Internal Medicine, Philadelphia, v. 131, n. 1, p. 7-13, jul. 1999.

FANG, J.; ALDERMAN, M. H. Serum Uric Acid and Cardiovascular Mortality: The NHANES I epidemiologic follow-up study, 1971-1992. Journal of the American Medical Association, Chicago, v. 283, n. 18, p. 2404-2410, 2000.

FARQUHARSON, C. A. J.; BUTLER, R.; HILL, A.; BELCH, J. J.; STRUTHERS, A. D. Allopurinol Improves endothelial dysfunction in chronic heart failure. Circulation, Baltimore, v. 106, n. 2, p. 221-226, jul. 2002.

FEIG, D. I.; KANG, D.; JOHNSON, R. J. Uric acid and cardiovascular risk. The New England Journal of Medicine, Boston, v. 359, n. 17, p. 1811-21, oct. 2008.

GRASSI, D.; FERRI, L.; DESIDERI, G.; DI GIOSIA, P.; CHELI, P.; DEL PINTO, R.; PROPERZI, G.; FERRI, C. Chronic hyperuricemia, uric acid deposit and cardiovascular Risk. Current Pharmaceutical Design, San Francisco, v. 19, n. 13, p. 2432-8, mar. 2013.

HARE, J. M.; MANGAL, B.; BROWN, J.; FISHER, C.; FREUDENBERGER, R.; COLUCCI, W. S.; MANN, D. L.; LIU, P.; GIVERTZ, M. M.; SCHWARZ, R. P. Impact of oxypurinol in patients with symptomatic heart failure. Results of the OPT-CHF study. Journal of The American College of Cardiology, New York, v. 51, n. 24, p. 2301-9, jun. 2008.

KANELLIS, J.; FEIG, D. I.; JOHNSON, R. J. Does asymptomatic hyperuricaemia contribute to the development of renal and cardiovascular disease? Nephrology, Melbourne, v. 9, n. 6, p. 394-9, dec. 2004.
KASAPKARA, H. A.; TOPSAKAL, R.; YARLIOGLUES, M.; YARLIOGLUES, G.; DOGDU, O.; ARDIC, I.; AKPEK, M.; KAYA, M. G. Effects of serum uric acid levels on coronary collateral circulation in patients with non-ST elevation acute coronary syndrome. Coronary Artery Disease, Philadelphia, v. 23, n. 7, p. 421-5, nov. 2012.

KAUFMAN, M.; GUGLIN, M. Uric acid in heart failure: a biomarker or therapeutic target? Heart Failure Reviews, Norwell, v. 18, n. 2, p. 177-86, mar. 2013.

KIVITY, S.; KOPEL, E.; MAOR, E.; ABUBACHAR, F.; SEGEV, S.; SIDI, Y.; OLCHOVSKY, D. Association of serum uric acid and cardiovascular disease in healthy adults. The American Journal of Cardiology, New York, v. 111, n. 8, p. 1146-51, apr. 2013.

KRISHNAN, E.; PANDYA, B. J.; LINGALAL, B.; HARIRI, A.; DABBOUS, O. Hyperuricemia and untreated gout are poor prognostic markers among those with a recent acute myocardial infarction. Arthritis Research \& Therapy, London, v. 14, n. 1, jan. 2012.

KRISHNAN, E.; SOKOLOVE, J. Uric acid in heart disease: a new C-reactive protein? Current Opinion in Rheumatology, Philadelphia, v. 23, n. 2, p. 174-7, mar. 2011.

NAGAHAMA, K.; INOUE, T.; ISEKI, K.; TOUMA, T.; KINJO, K.; OHYA, Y.; TAKISHITA, $\mathrm{S}$. Hyperuricemia as a predictor of hypertension in a screened cohort in Okinawa Japan. Hypertension research: official journal of the Japanese Society of Hypertension, Toyonaka, v. 27, n. 11, p. 835-41, nov. 2004.

NASR, G.; MAURICE, C. Allopurinol and global left myocardial function in heart failure patients. Journal of Cardiovascular Disease Research, Mumbai, v. 1, n. 4, p. 191-5, oct. 2010.

NDREPEPA, G.; BRAUN, S.; KING, L.; CASSESE, S.; TADA, T.; FUSARO, M.; HADAMITZKY, M.; HAASE H. U.; SCHÖMIG, A.; KASTRATI, A. Prognostic value of uric acid in patients with Type 2 diabetes mellitus and coronary artery disease. Clinical Science, London, v. 124, n. 4, p. 259-68, feb. 2013. 
NISKANEN, L. K.; LAAKSONEN, D. E.; NYYSONEN, K.; ALFTHAN, G.; LAKKA, H. M.; LAKKA, T. A. Uric Acid Level as a risk factor for cardiovascular and all-cause mortality in middle-aged men. Archives of Internal Medicine, Chicago, v. 164, n. 14, p. 1546-51, jul. 2014.

OIKONEN,M.;WENDELIN,S.M.;LYYTIKÄINEN, L. P.; SIITONEN, N.; LOO, B. M.; JULA, A.; SEPPÄLÄ, I.; SAARIKOSKI, L.; LEHTIMÄKI, T.; HUTRI-KÄHÖNEN, N.; JUONALA, M.; KÄHÖNEN, M.; HUUPPONEN, R.; VIIKARI, J. S.; RAITAKARI,O. T. Associations between serum uric acid and markers of subclinical atherosclerosis in young adults. The cardiovascular risk in Young Finns study. Atherosclerosis, Amsterdam, v. 223, n. 2, p. 497-503, aug. 2012.

PACIFICO, L.; CANTISAN, V.; ANANIA, C.; BONAIUTO, E.; MARTINO, F.; PASCONE, R.; CHIESA, C. Serum uric acid and its association with metabolic syndrome and carotid atherosclerosis in obese children. European Journal of Endocrinology, Oslo, v. 160, n. 1, p. 45-52, jan. 2009.

SIMÃO, A. N. C.; DICHI, J. B.; BARBOSA, D. S.; CECCHINI, R.; DICHI, I. Influence of UA and gamma-glutamyltransferase on total antioxidant capacity and oxidative stress in patients with MS. Nutrition, Burbank, v. 24, n. 7/8, p. 675-81, jul-ago. 2008.

SIMÃO, A. N. C.; LOZOVOY, M. A.; DICHI, I. The uric acid metabolism pathway as a therapeutic target in hyperuricemia related to metabolic syndrome. Expert Opinion on Therapeutic Targets. London, v. 16, n. 12, p. 1175-1187, dec. 2012.

TANIGUCHI, Y.; HAYASHI, T.; TSUMURA, K.; ENDO, G.; FUJII, S.; OKADA, K. Serum uric acid and the risk for hypertension and Type 2 diabetes in Japanese men: the Osaka Health Survey. Journal of Hypertension, London,v. 19, n. 7, p. 1209-15, jul. 2001.

UYSAL, O. K.; SAHIN,D, Y.; DURAN, M.; TURKOGLU, C.; YILDIRIM, A.; ELBASAN, Z.; OZKAN, B.; TEKIN, K.; KUNAK, A. U.; YILMAZ, Y.; KAYA, M. G.; GUR, M.; CAYLI, M. Association between uric acid and coronary collateral circulation in patients with stable coronary artery disease, Angiology, New York, v. 65, n. 3, p. 227-31, mar. 2014.
VENTURINI, D.; SIMÃO, A.N.C.; SCRIPES, N. A.; BAHLS, L. D.; MELO, P. A. S.; BELINETTI, F. M., LOZOVOY, M. A. B.; DICHI, I. Evaluation of oxidative stress in overweight subjects with or without metabolic syndrome. Obesity, Malden, v. 20, n. 12, p. 2361-6, dec. 2012.

WANNAMETHEE, S. G.; SHAPER, A. G.; WHINCUP, P. H. Serum urate and the risk of major coronary heart disease events. Heart, London, v. 78, n. 2, p. 147-53, aug. 1997.

WHEELER, J. G.; JUZWISHIN, K. D. M.; EIRIKSDOTTIR, G.; GUDNASON, V.; DANESH, J. Serum uric acid and coronary heart disease in 9,458 incident cases and 155,084 controls: prospective study and meta-analysis. PLos Medicine, San Francisco, v. 2, n. 3, p. e76, mar. 2005.

WILDI, K.; HAAF, P.; REICHLIN, T.; ACEMOGLU, R.; SCHNEIDER, J.; BALMELLI, C.; DREXLER, B.; TWERENBOLD, R.; MOSIMANN, T.; REITER, M.; MUELLER, M.; ERNST, S.; BALLARINO, P.; ZELLWEGER, C.; MOEHRING, B.; VILAPLANA, C.; FREIDANK, H.; MUELLER, C. Uric acid for diagnosis and risk stratification in suspected myocardial infarction. European journal of clinical investigation. European Journal of Clinical Investigation, Berlin, v. 43, n. 2, p. 174-82, feb. 2013.

YANIK, M.; FEIG, D. I. Serum urate: a biomarker or treatment target in pediatric hypertension? Current Opinion in Cardiology, London, v. 28, n. 4, p. 433-8, jul. 2013. 